\title{
An Mobile Android Voice Driven Supervisory System for an Experimental Multi-Tasking Relay Process
}

\author{
P.Mariappan \\ Professor, Department of ECE, S V College of Engineering, Tirupati, A.P, India
}

\begin{abstract}
More current solid fluid administrations are developing in an extremely fast stage yet there are issues which are left unsolved. One such issue is controlling a real time process in profoundly inflammable environment like naphtha stream funnel, Jet fuel stockpiling compartments and so on. One such answer for the above issue is remote control of the stream valve utilizing GSM handset circuit started through voice. The parameters are checked by microcontroller and send the short message service to client in the event of any variations from the normal. The client if wishes can control the framework by a voice charge through android telephone.
\end{abstract}

KEYWORDS: GSM transceiver circuit, Flow valve, Android Phone, Remote control

\section{INTRODUCTION}

Industry has experienced different improvements. As the advancements expand, the requirement for controlling parameters and sensing it in an effective and real time manner [8] turns into a need of great importance. Overshooting the edges for these parameters will lead to disastrous consequences. The controlling operation includes embrace capita venture and involves gigantic physical space for controlling operation. Consolidating the qualities of the sensor [4] with the comfort of cellular telephones, we can build up a mixture of novel applications in the portable terminal, which get to be handheld stimulation for individuals in extra time, and can give designers with new ranges for improvement. Consequently, the controlling operation in a productive and financially savvy strategy in this paper is done utilizing GSM modem and an Android Application.

For this, the proposed framework utilizes the stream sensor for controlling the stream of fluid, Solenoid valve for controlling the gulf and the temperature sensor LM35 for checking the temperature. The sensors are interfaced with MSP430 Microcontroller through one of the ADC ports. Utilizing a GSM SIM 900A modem, gadgets can be controlled in a productive way from remote areas [9]. The entire controlling procedure is done utilizing Android application .The voice to content empowered application is utilized to send a short informing support of GSM modem which will control the machine associated with it[10].

The execution of the above framework happens in two ways. Both the ways utilize an android application created utilizing Android Developer studio and Java SDK Manager [1]. The primary system is the programmed mode and the second technique is the manual mode. The Manual mode comprises of an Android Voice to content transformation module included. As the speaker gives in the voice charge, the relating content would be sent to telephone number determined. This content would be perceived by the GSM modem and it is imparted to microcontroller correspondingly. The microcontroller performs the controlling choices in like manner.

\section{ANDROID APPLICATION DEVELOPMENT}

Android is a Linux based Operating framework [1] grew by Google in a joint effort with Open Handset Alliance to create Android Applications. Linux kernel-based architecture model adds the use of Linux to the mobile industry, taking advantage of the knowledge and features offered by Linux [3]. The Android platform consists of several layers which provide a complete software stack. Android applications are Java-based and this factor entails the use of a virtual machine VM environment, with its advantages. The application improvement includes introducing the Java Development Kit known as SDK. Android SDK gives the important Application Programmable Interface needed for creating android applications utilizing Java. Android has an implicit web-pack for creating webprogram applications.

Subsequent to introducing the SDK record, we can access these tools through ADT plug-in right from our Eclipse IDE, or from the command line. Eclipse IDE is the favored system for advancement as it invokes every one of the libraries and tools included for complete development. 


\section{International Journal of Innovative Research in Science, Engineering and Technology}

Vol. 1, Issue 2, December 2012

\section{A. DEVELOPMENT PROCESS IN ANDROID}

The fundamental strides in creating android applications are indicated in Figure 1.The development steps resemble the four development phases [2], which incorporate the accompanying:

In the setup phase, we set up the environment needed for developing an android application by installing the software. This includes introducing Eclipse IDE, JDK and related records on the framework. Subsequent to introducing the JDK document, it is crucial to build an Android Virtual Device taking into account the JDK file.

Next phase is the development, in which, we setup the undertaking with the important assets. This includes android level determination, android virtual device suited for our application and indicating the base android adaptation needed for running the custom application.

Troubleshooting and Testing is done utilizing android

improvement studio tool which is the following stage in the life cycle. The debugging involves creating an .apk document made by compiler.

Publishing phase involves building our application after configuring and distribute to users through Google Play store or use it for individual purposes.

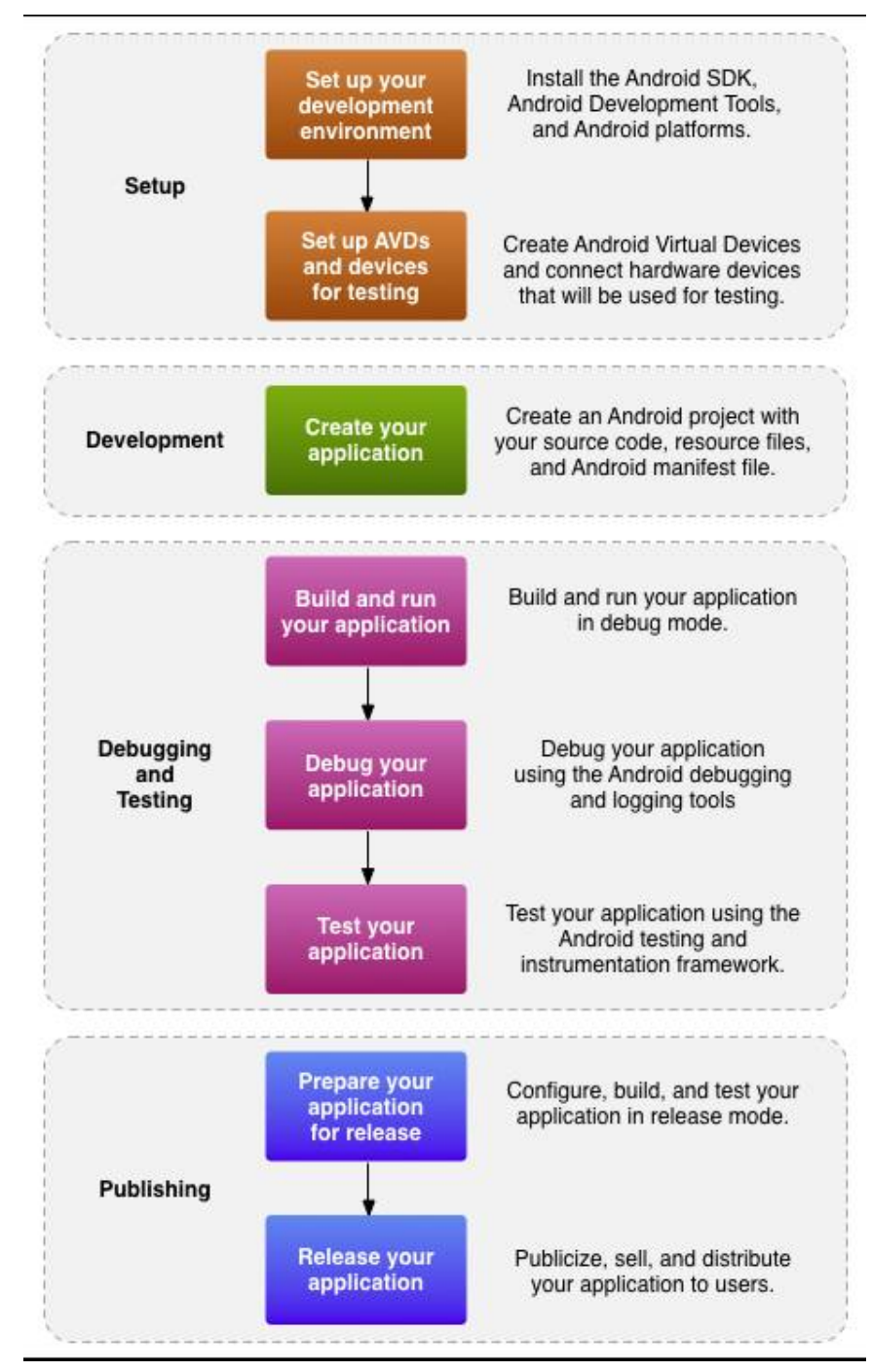

Fig 1: Android Development Cycle

\section{B. ANDROID APPLICATION CLASSIFICATION}

Figure 2 demonstrates the Android OS Structure, An Android application comprises of numerous classifications [2]; few of them are activities, services, content providers and broadcast receivers.

Activity is the visible client interface appeared when another undertaking is begun in the android improvement studio. The application interface (E.g Buttons, Text perspective) is produced utilizing xml. [6] The xml documents gets interlinked utilizing java environment.

Any application that obliges upgrade for any 


\section{International Journal of Innovative Research in Science, Engineering and Technology}

Vol. 1, Issue 2, December 2012

application ought to utilize service. These incorporate system connectors for overhauling the application and legitimate android environment for running the redesigned application.

Content providers are the means of accessing the larger data involving android application. For example, SQL data access by an application. In Applications which involves larger data such as Voice to text conversions, this content provider is used.

Broadcast receivers are used for a respond to an event (E.g. receipt of a text message). This involves Java scripts for broadcasts. The broadcasts in android are called as toast.

\section{SPEECH TO TEXT CONVERSION}

It is the method for getting to the bigger data involving android application. Case in point, SQL information access by an application. In Applications which includes bigger information, for example, Voice to text transformations, this content provider is utilized as shown in Figure 2.

\begin{tabular}{|c|}
\hline Application \\
\hline Application Framework \\
\hline Libraries \\
Android Routine \\
\hline Linux Kernel \\
\end{tabular}

Fig 2: Android OS Structure

Figure 3 shows the custom android application for relay controlling with Google Voice Search. The Voice to text conversion system involves processing the speech signal after noise cancellation. The noise cancelled signal is then used for conversion. Speech to text conversion is the ability of the application to recognize the speech signal given in any language and correspondingly generate a text based on the database for that particular language. 


\section{International Journal of Innovative Research in Science, Engineering and Technology}

Vol. 1, Issue 2, December 2012

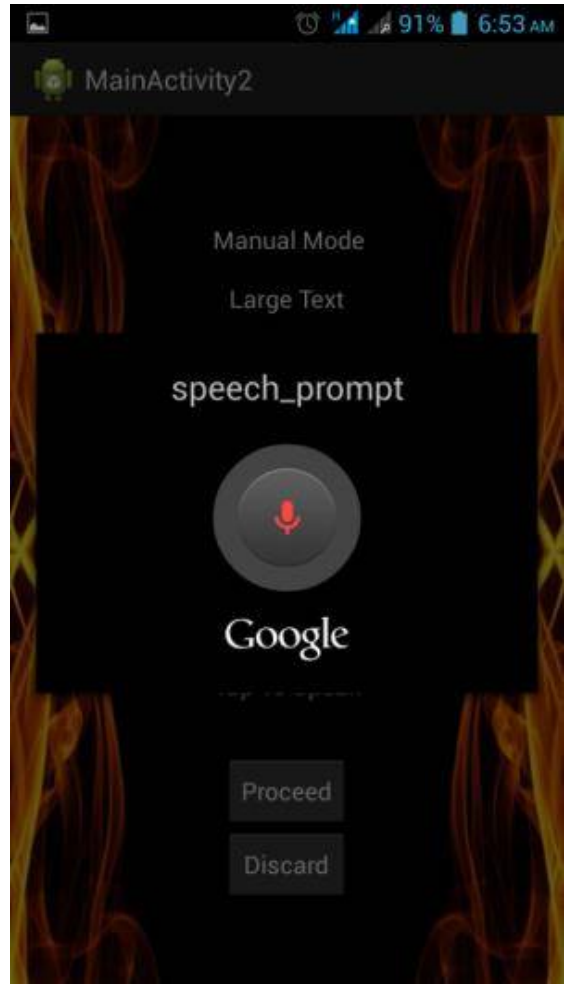

Fig 3: Custom Android Application for relay controlling with Google's Voice Search

The Speech to Text conversion system employed in our project consists of following elements such as acquiring Speech, converting speech to text and transmitting SMS.

Speech Acquisition is an indispensable part of Speech Acquisition gadget. This includes acquiring the signal after noise abrogation. This signal is then handled to create suitable text content. Sphinx structure is utilized for preprocessing the speech command. Sphinx, which is profoundly dependable in configuration, is likewise very adaptable and measured. There are three modules in the sphinx casing work frontend decoder, Linguist Front end takes the input speech signals and parameterized it into sequence of features. The Linguist interprets any kind of standard dialect model, alongside articulation data from the Dictionary and basic data from one or more arrangements of Acoustic Models, into a Search Graph. The Search Manager in the Decoder uses the Features from the Front End and the Search Graph from the Linguist to perform the real translating, creating Results.

The significant obstacle in making voice to content change is the database. It is crucial to have a colossal database for making this voice to content as a worldwide application. The frequencies of clients in the same dialect will be distinctive and consequently it is all that much

fundamental that we must have a database. Consequently we have to utilize database which is as of now given by "Google". On the off chance that we attempt to make the database physically it will make time and space issue. To make such an immense database part of time will west and this task is for portable clients whose interior memory of versatile is for the most part low and the database for this is framework is all that much colossal. As opposed to making a database for this framework we have to utilize existing database which is accessible at the web server. The discourse data is gathered in the holder and the send it to the peach and recurrence correlation the acknowledgment occur. To get right content which is talked we need preparing on data document for that neural system (NN) is utilized[5] and the yield is gathered in the content record and SMS is prepared to send.

The next step is SMS Transmission, during SMS sending stage the speech samples which is changed over into text content is send as a SMS to the next gadget. In this stage two android emulators are utilized to send and get the SMS. From the first emulator which we consider as portable, one sends the message and other gadget get it at the inbox. Here we utilize Android Manifest.xml to include the two consents - SEND_SMS and RECEIVE_SMS. Next we have added here main.xml to get the telephone number of sender. Next, in the SMS action, we wire up the Button so that when the client taps on it, we will verify that the telephone number of the beneficiary and the message is entered before we send the message utilizing the sendSMS() capacity, which we will characterize in no time. To send a SMS message, we utilize the SmsManager class. At that point we will call the getDefault () static system to acquire a SMS Manager item.

The sendTextMessage () system sends the SMS message with a Pending Intent. The Pending Intent article is utilized to recognize an objective to conjure at a later time. Case in point, subsequent to sending the 


\section{International Journal of Innovative Research in Science, Engineering and Technology}

Vol. 1, Issue 2, December 2012

message, you can utilize a Pending Intent item to show another action. For this situation, the Pending Intent object (pi) is just indicating the same action (SMS.java), so when the SMS is sent, nothing will happen. At the point when a SMS message is sent, the first Broadcast Receiver's on Receive occasion will fire. This is the place we check the status of the sending procedure. The second Pending Intent item (conveyed PI) screens the conveyance process. The second Broadcast Receiver's on Receive occasion will fire when a SMS is effectively conveyed.

\section{GSM SIM 900A}

A GSM modem is a specific sort of modem which recognizes a SIM card, and works more than participation to a convenient executive, much the same as a cell phone. From the adaptable overseer perspective, a GSM modem looks basically like a phone. Exactly when a GSM modem is connected with a PC, this allows the PC to use the GSM modem to give over the convenient framework. While these GSM modems are most routinely used to give compact web joining, most of them can similarly be used for sending and getting SMS and MMS messages. GSM modem must support a "connected AT request set" for sending/getting SMS messages. GSM modems can be an energetic and capable way to deal with start with SMS, in light of the fact that an unprecedented participation to a SMS organization supplier is not required. In numerous parts of the world, GSM modems are a sagacious response for receiving SMS messages, in light of the way that the sender is paying for the message transport. To begin, insert a GSM SIM card into the modem and unite it to an open USB port on your PC.

AT Commands are used in GSM modem for accessingthe data's and sending the data.AT means Attention.Every command line starts with "AT".

These are of two types of AT commands in GSM Modem: Basic and Extended.

- $\quad \mathrm{ATEO}=$ Echo off

- $\quad$ ATE1 = Echo on

- $\quad$ ATD = Call a dial no.

- $\quad$ ATDL = Redial last telephone no.

- ATA = Answer an incoming call

- $\mathrm{ATH}=$ Disconnect existing connection

- $\mathrm{AT}+\mathrm{CMGS}=$ To send SMS Syntax:

- $\mathrm{AT}+\mathrm{CMGS}=$ = 9479555640"

- $\quad$ Press enter Type text and press ctrl+z

- $\mathrm{AT}+\mathrm{CMGR}=$ To read SMS

- Syntax : AT+ CMGR=1; Reads first SMS in sim card

- $\mathrm{AT}+\mathrm{CMGD}=$ To delete SMS Syntax: $\mathrm{AT}+\mathrm{CMGD}=1$;

- Deletes first SMS in sim card

\section{HARDWARE IMPLEMENTATION}

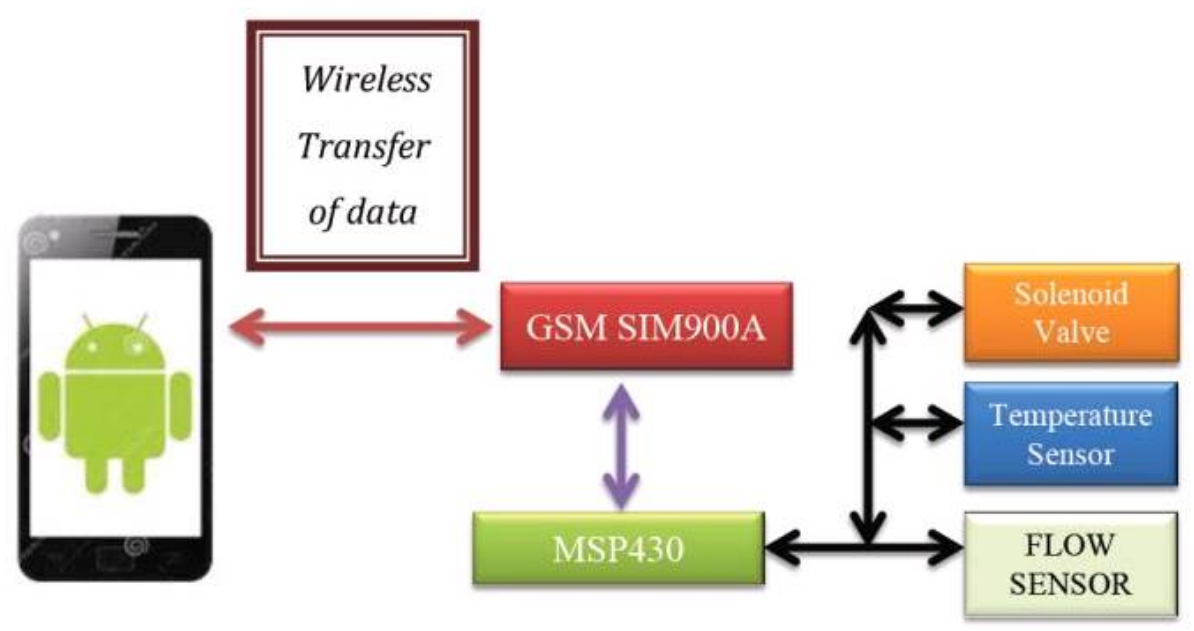

Fig 4: Block Representation of implemented real time systems

The equipment realization as shown in Figure 4 includes interfacing RS232 Port of GSM Modem to the 


\section{International Journal of Innovative Research in Science, Engineering and Technology}

Vol. 1, Issue 2, December 2012

MSP430 microcontroller utilizing MAX 232 IC. The MSP430 is used for low power controlled contraptions and the electric current pulled in unmoving mode can be under 1

$\mu \mathrm{A}$. The top CPU pace is $25 \mathrm{MHz}$ It can be throttled back for lower power utilization. The MSP430 additionally utilizes six distinctive low-control modes, which can cripple unneeded clocks and CPU. The device arrives in an assortment of designs highlighting the standard peripherals: internal oscillator, clock including PWM, watchdog, USART, SPI, I²C, 10/12/14/16/24-bit ADCs and brownout hardware.

The data from the Android telephone is associated with the MSP430 and this is utilized to control the solenoid valve. Solenoid is fundamentally a $12 \mathrm{~V}$ gadget utilized for controlling the water stream in a procedure. It fundamentally chips away at the standard of induction. At the point when the supply is given, the coil gets energized and it drives the relay coil as shown in Figure 7 which closes or opens the shutter taking into account the past state.

The stream of the fluid is observed utilizing a stream sensor. It is a device for distinguishing the rate of fluid stream. Ordinarily a stream sensor is the recognizing segment used as a piece of a stream meter, or stream logger, to record the surge of fluids. As is substantial for all sensors, precise precision of estimation obliges handiness for change. Stream sensor is a sort of Hall Effect sensor. It is a transducer that varies its yield voltage in light of an alluring field. Hall Effect sensors are used for closeness trading, arranging, speed disclosure, and current recognizing applications. In its minimum complex shape, the sensor acts as a straightforward transducer, straight forwardly giving back a voltage. With a known appealing field, its partition from the Hall plate can be determined. Using social occasions of sensors, the relative position of the magnet can be closed. Frequently, a Hall sensor is joined with equipment that allows the device to act in a mechanized (on/off) mode, and may be known as a switch in this game plan. The weight of the fluid can be checked after alignment. The alignment is done using the formula

Calculations for configuring Pressure sensor

- Resolution Voltage-Vref / $2^{\mathrm{n}}$

- $\mathrm{N}$ is the no channels of ADC

- Suppose the LM35 gives a value of $0.34 \mathrm{~V}$,we get the output as

$$
\text { - }(0.34) /\left(4.88 \times 10^{\wedge}-3\right)=69^{\circ} \mathrm{C} \text {. }
$$

- $\operatorname{Per}{ }^{\mathrm{O}} \mathrm{C}=0.08 \mathrm{~V}$

- For $30^{0} \mathrm{~V}=2.4 \mathrm{~V}$

- 2.4 / $\left(4.88 \times 10^{\wedge}-3\right)=491$

- This value should be loaded in the code for setting the thresholds.

\section{ALGORITHM}

The figure describes the steps involved in extracting the message from the SIM 900A modem after receiving. Retrieving the message involves the following steps

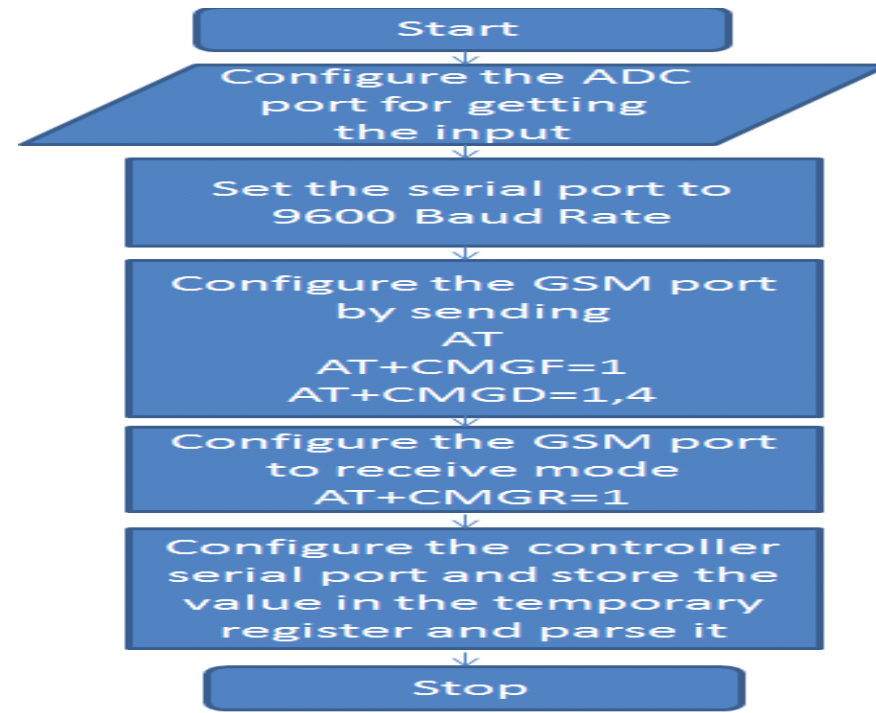

Fig 5: Flow Chart for controlling the relay switching using microcontroller 
International Journal of Innovative Research in Science, Engineering and Technology Vol. 1, Issue 2, December 2012

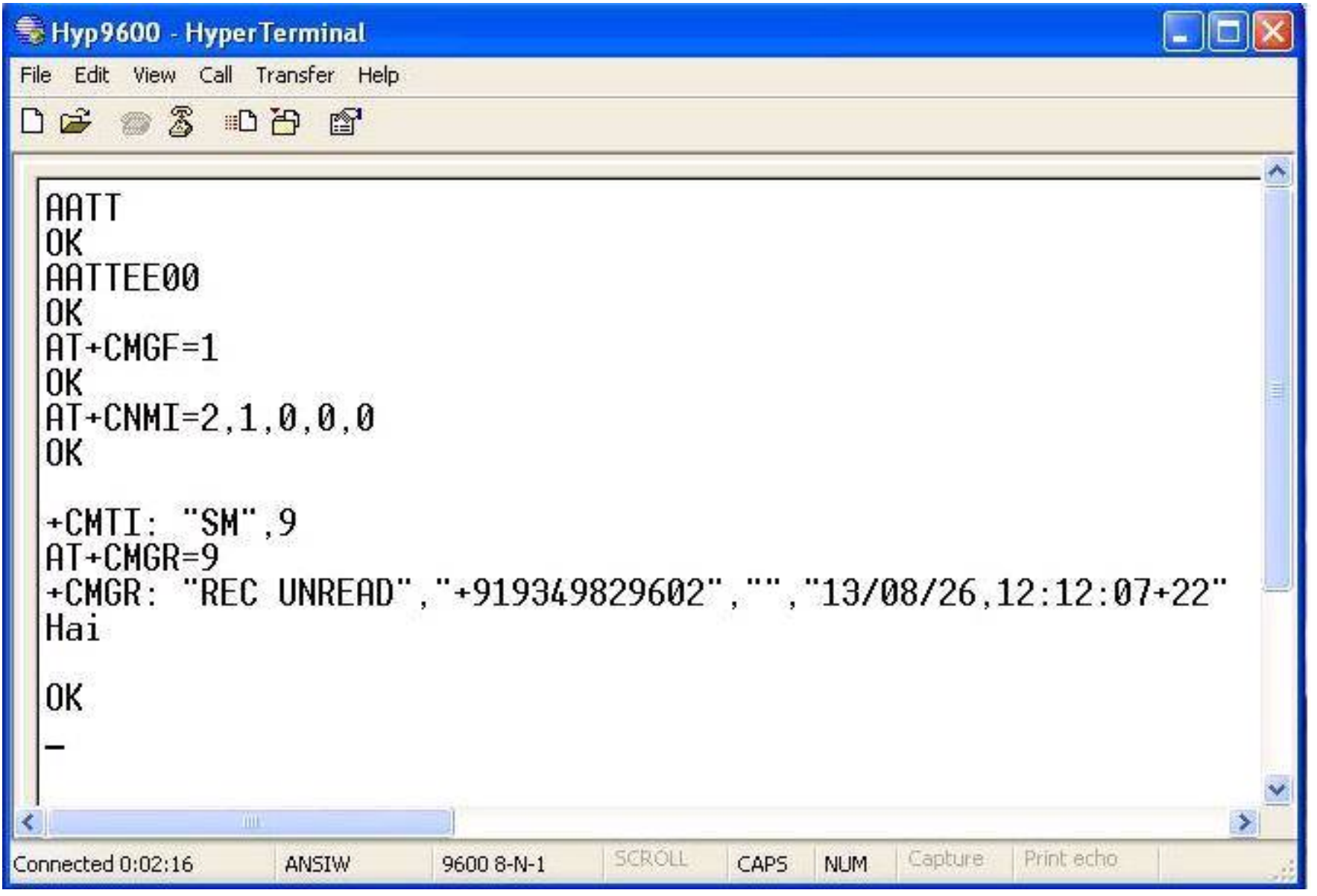

Fig 6: AT Commands for configuring the GSM Modem

\section{RESULTS AND DISCUSSUION}

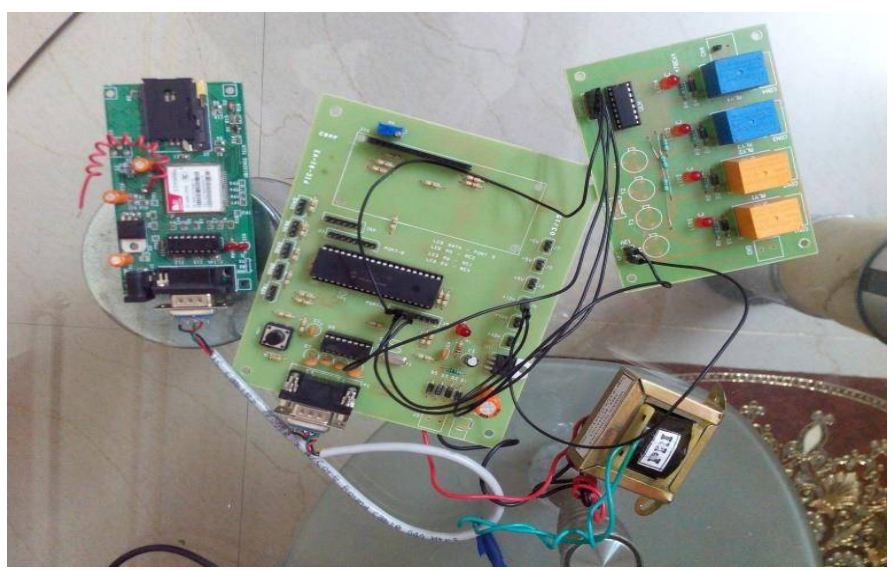

Fig 7: Hardware Setup of the implemented real time system 


\section{International Journal of Innovative Research in Science, Engineering and Technology} Vol. 1, Issue 2, December 2012

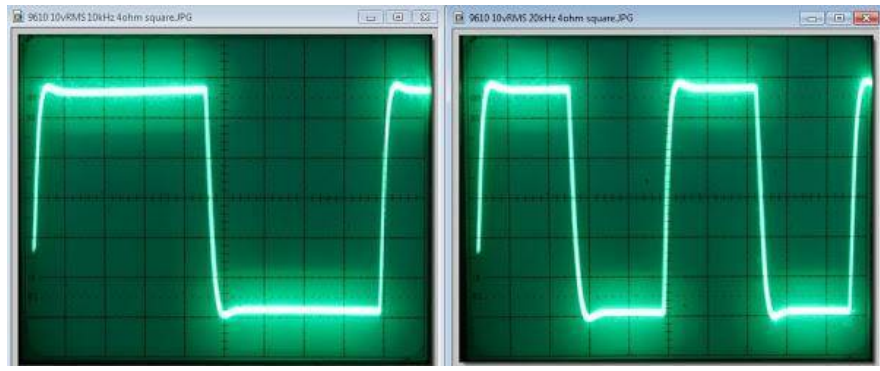

Fig 8: Voice driven analog Switching pattern on CRO

The hardware result from CRO is demonstrated using cathode ray oscilloscope. The output from the MSP430 is given to the relay circuit .The relay circuit output is given to a CRO output. The output goes to output high $(5 \mathrm{v})$ and this goes low when the relay switches OFF. The relay output is coupled to solenoid valve and peripherals so that the output can be controlled.

\section{CONCLUSION}

The system makes productive utilization of the most recent innovation, to help the commercial enterprises in their regular control of real time systems. It can be thought about as a solution for the hardships confronted by the industries now-a-days, since it gives a real time control in industrial environment. It has a global system for mobile communication unit, having a supporter number through a sim card which shapes a connection between the control process and the mobile unit. Henceforth, the system changes over the voice command to message and taking into account order, proper control procedure is controlled. The GSM technology is used in light of its principle highlights which

can be recorded as, complete openness, straightforwardness, littler measure of sign decay, expansive scope territory and so on. every one of these components improve this innovation a possibility for sending control commands and accepting overhauls from a remote spot. Further, the execution investigation of the framework is assessed by cathode ray oscilloscope. Thus, it can be inferred that the proposed system is practical and includes least inactivity. The paper can be further reached out for monitoring and controlling the real time system with the existing framework.

\section{REFERENCES}

[1] Ma, Li, Lei Gu, and Jin e. "Research and Development of Mobile Application for android Platform." (2014). [2]Garima Pandey, Diksha Dani."Android Mobile Application Build on Eclipse". International Journal of Scientific and Research Publications, Volume 4, Issue 2, February 20141 ISSN 2250-3153

[3] Shyam Bhati, Sandeep Sharma, Karan Singh.” Review On Google Android a Mobile Platform.” IOSR Journal of Computer Engineering (IOSRJCE) e-ISSN: 2278-0661, p- ISSN: 2278-8727Volume 10, Issue 5 (Mar. - Apr. 2013), PP

21-25

[4] Zhi-An, Yi, and Mu Chun-Miao. "The development and application of sensor based on android." Information Science and Digital Content Technology (ICIDT), 2012 8th International Conference on. Vol. 1. IEEE, 2012.

[5] Falas T, Stafylopatis, A, A-G "Neural Networks in text- to-speech systems for the Greek language" Electro technical Conference, 2000.MELECON 200.10 ${ }^{\text {th }}$ Mediterranean (Volume 2)

[6] Pallavi Raut and Satyaveer Tomar. "Android Mobile

Automation Framework" IJECS Volume 3 Issue 10

October, 2014 Page No.8555-8560

[7] Anna Zhu, Peijie Lin and Shuying Cheng ." Design and Appliances Control System based on the Android Smartphone" Control engineering and Communication Technology (ICCECT), 2012 International Conference on, September, Page No 56-59

[8] Szakacs-Simon, P.S.A.; Perniu, L."Android application developed to extend health monitoring device range and real-time patient tracking" Computational Cybernetics (ICCC), 2013 IEEE 9th International Conference on 8-10

July $2013171-175$

[9] Young-Hoon Jeon, Hyunsik Ahn "Smart-Phone Based Interface for Mobile Robot Control" The Institute of Electronics Engineers of Korea 2010 Annual Summer Conference, Vol. 33, No. 1, pp. 1951-1953, 2010.

[10] Mhapsekar, A.; Kulkarni, P.; Nagarsekar, U.; Kalbande "Voice enabled Android application for vehicular complaint system: Using GPS and GSM-SMS technology"D.R.Information and Communication Technologies (WICT), 2012 World Congress on 2012, Pages: 520 - 524, DOI: 10.1109/WICT.2012.6409133. 